\title{
Neural network differentiation of optic neuritis and anterior ischaemic optic neuropathy
}

\author{
Leonard A Levin, Joseph F Rizzo III, Simmons Lessell
}

\begin{abstract}
Aims-The efficacy of an artificial intelligence technique, neural network analysis, was examined in differentiating two optic neuropathies with overlapping clinical profiles-idiopathic optic neuritis (ON) and non-arteritic anterior ischaemic optic neuropathy (AION).

Methods-A neural network was trained with data from 116 patients with 'gold standard' diagnoses of ON or AION. It was then tested with data from 128 patients with presumed ON or AION, and the correlation of the network's diagnosis with that of expert clinicians tabulated.

Results-The network agreed with the clinicians on $97.8 \%$ (88 of 90 ) of the patients with presumed ON and $94.7 \%$ (36 of 38) of the patients with presumed AION. Youth, female sex, better initial acuity, a central scotoma, subsequent improvement in acuity, or progressive disease biased the network towards a diagnosis of $O N$, while advanced age, male sex, presence of hypertension, poor initial acuity, an altitudinal field defect, disc oedema, or less improvement in acuity biased the network towards a diagnosis of AION.

Conclusion-Neural network analysis is a useful technique for classification of optic neuropathies, particularly where there is overlap of clinical findings.

(Br f Ophthalmol 1996;80:835-839)
\end{abstract}

Idiopathic optic neuritis (ON) and nonarteritic anterior ischaemic optic neuropathy (AION) are acute optic neuropathies with overlapping clinical profiles. ON typically presents as painful loss of vision in younger patients and has a good potential for improvement, whereas AION typically presents as painless loss of vision in the middle aged or elderly with usually less recovery. Although in many cases the diagnosis of these optic neuropathies is straightforward, neither the presence of pain, age of the patient, nor a variety of other features can confidently distinguish between the two disorders. ${ }^{1}$

Neural network analysis is an artificial intelligence technique for the classification of groups, and thus is well suited to computer aided diagnosis. The neural network itself is a simulation of a set of neurons, organised in layers (Fig 1). The advantages of neural networks for classification, as well as rationales for its application, are well summarised in a recent application to classifying automated perimetry visual fields. ${ }^{2}$ We describe here the use of neu- ral network analysis to help differentiate between $\mathrm{ON}$ and $\mathrm{AION}$, and to generate guidelines for distinguishing these conditions when they overlap clinically.

\section{Materials and methods}

PATIENT SELECTION CRITERIA

Patient records from the neuro-ophthalmology database at the Massachusetts Eye and Ear Infirmary were analysed retrospectively. All of the patients were described previously in a study of overlapping profiles of $\mathrm{ON}$ and AION, ${ }^{1}$ with some excluded because of insufficient data on the initial or follow up visits. Details of the clinical examination were as described in that reference. Every patient given a clinical diagnosis of either ON or AION by one of two experienced neuroophthalmologists (JR or SL), seen within 2 weeks of presentation, and followed for at least 4 months until stabilisation of visual function was selected. The diagnosis was one based purely on clinical impression, and not on any particular criteria. Patients with hereditary disorders, temporal arteritis, or other granulomatous inflammations or vasculitides, infection, or traumatic optic neuropathy were excluded.

There were 244 patients with optic neuropathies clinically diagnosed as ON or AION, and these were divided into two groups. Firstly, a group of 116 patients with 'gold standard' diagnoses was defined retrospectively (Fig 2). Specifically, a 'gold standard' diagnosis of ON was assigned when the patient subsequently developed multiple sclerosis; 69 patients met this criterion. A gold standard diagnosis of AION was assigned when the patient had no pain, was over the age of 60 , and had disc oedema; 47 patients met these criteria. These two definitions were consistent with the 'gold standard criteria' of Rizzo and Lessell.' The gold standard groups of patients was used to train a neural network (see below).

The remaining 128 patients (90 patients with presumed $O N$ and 38 patients with presumed AION) formed a second group, which was subsequently used to test how often the network diagnosis matched the diagnosis of the expert clinician. These patients did not meet the gold standard criteria enumerated above, and their diagnosis was based simply on clinical impression at the time of first examination by the examining neuro-ophthalmologist, and not on any specific criteria.

NEURAL NETWORK DESIGN

The majority of neural networks contain three layers of neurons, and a similar format was 
Table 1 Clinical variables

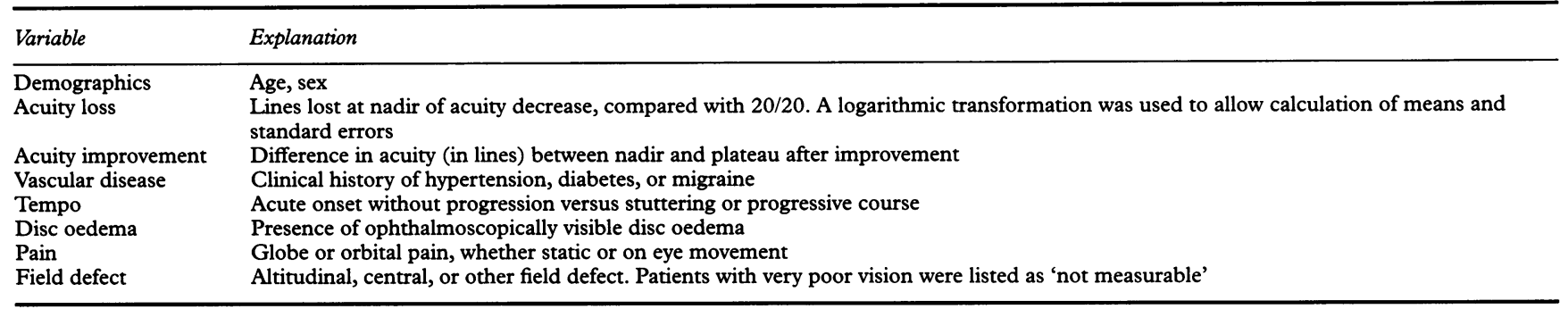

used for the network in this study (see Fig 1). Clinical and other data enter into the input layer, where each neuron corresponds to a specific clinical item (for example, age, visual acuity, etc). Each input neuron is connected to neurons in a second layer, called the hidden layer, and each of these connect to neurons in a third and final layer, the output layer. The value of each neuron in the hidden layer is calculated by multiplying the value of each neuron to which it is connected by the strength of the connection and then applying a function to reduce the effect of extreme values. Similarly, each neuron in the output layer derives its value from the neurons in the hidden layer to which it is connected. The strengths of the connections are determined in the training

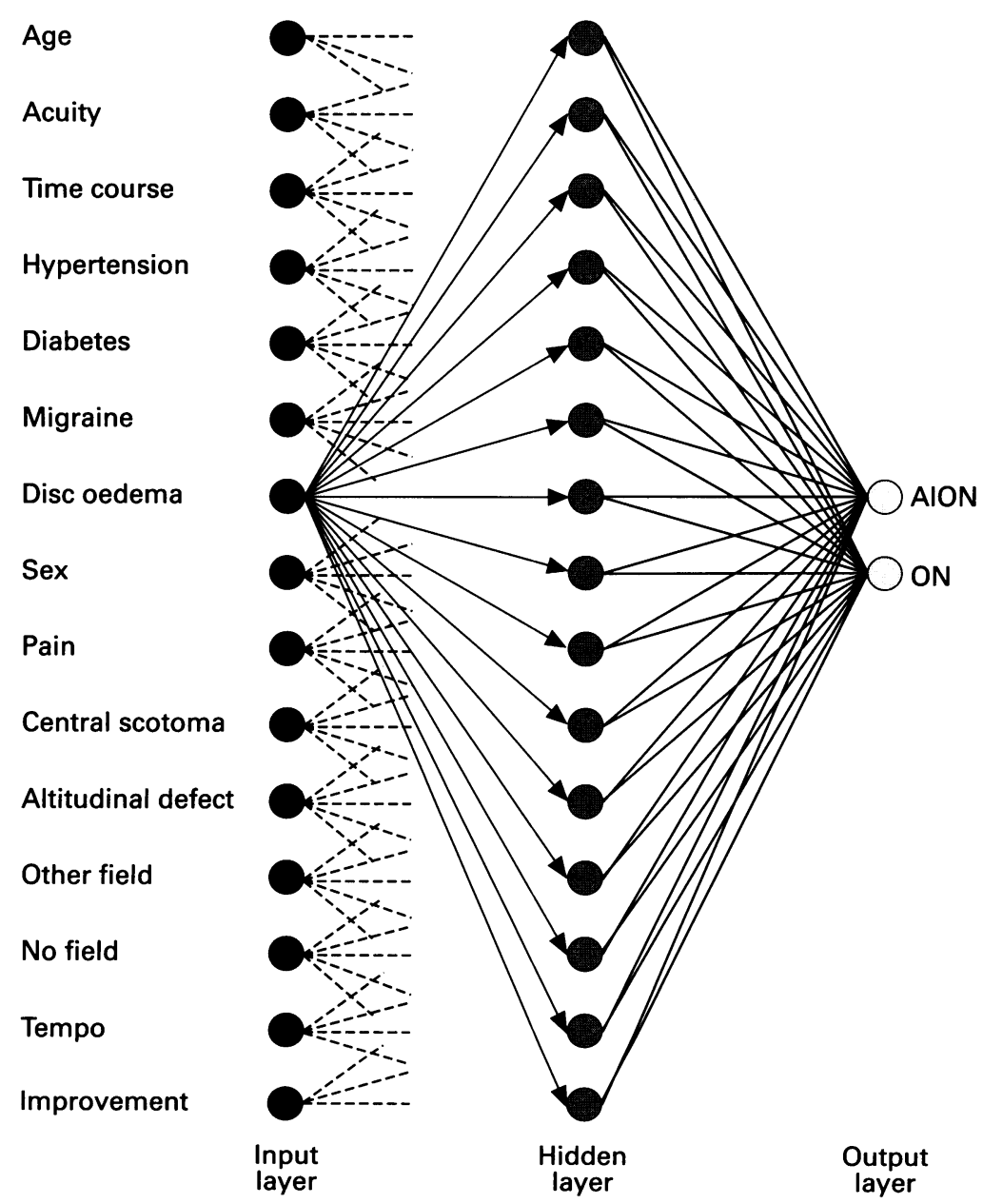

Figure 1 Schematic representation of the neural network. Clinical variables enter from the left and are fed to simulated neurons in the input layer, thus determining their value. The values of the neurons in the hidden layer depend on the values of the input neurons, weighted by the strength of their connections. These weightings are established when the neural network is 'trained'. The values of the output neurons are determined by the values of the hidden layer neurons in a similar fashion. The relative value (whichever is greater) of the output neurons represents the network's preferred diagnosis. process using a computer algorithm ${ }^{3}$; once complete, the network can be used to diagnose new patients by applying the clinical data to the input neurons, and recording the values from the output neurons.

\section{NEURAL NETWORK TRAINING}

For each patient with either gold standard ON or gold standard AION, the items listed in Table 1 were tabulated and used as input to the neural network. All inputs were multiplied by a factor so that they ranged from 0.0 to 1.0 (for example, the patient's age was divided by 100). The neural network was simulated with 15 input neurons, corresponding with each of the items in Table 1. Each of these 15 input neurons connected to each of 15 hidden layer neurons (making $15 \times 15=225$ connections), and each of the 15 hidden layer neurons connected to two output layer neurons (Fig 1). These two output neurons corresponded to the diagnoses of $\mathrm{ON}$ and AION, respectively.

In random sequence the clinical data from each gold standard patient were used as inputs to the input neurons, thus activating the network. At the same time the difference between the network's output neurons (representing the calculated diagnosis) and the actual diagnosis was used to calculate an error value; this error value was then used to modify the strengths of connections of the various network neurons. This technique, called back propagation learning, ${ }^{23}$ was performed until the total squared deviations between the predicted and actual diagnoses reached a preset bound of 0.005 .

NEURAL NETWORK TESTING

At this point the network was considered to have 'learned' the gold standard patient data. The network was then presented with data from the non-gold standard patients, to determine whether the network would agree with the expert clinician. A diagnosis of $\mathrm{ON}$ by the network was recorded if the ON neuron in the output layer had a greater value than the AION neuron, and vice versa. The agreement between the diagnosis calculated by the network and the clinical diagnosis of the expert clinicians was tabulated in a $2 \times 2$ table. Patient diagnoses where the network and the clinician agreed were termed concordant, while cases where there was disagreement were termed discordant.

The importance of each clinical datum (for example, age, sex, etc) in discriminating the two disorders was studied in a second set of simulations. This was done by varying each 
Table 2 Differences in clinical criteria of the patient groups

\begin{tabular}{lllll}
\hline & \multicolumn{2}{l}{ Group } & & \\
\cline { 2 - 5 } & $I$ (concordant) & II (discordant) & III (concordant) & $I V$ (discordant) \\
\hline Clinical diagnosis & ON & ON & AION & AION \\
Network diagnosis & ON & AION & AION & ON \\
Number of patients & 88 & 2 & 36 & 2 \\
Age (years) & $32.5(1.0)$ & 39,47 & $55.8(1.6)$ & 42,79 \\
Acuity loss & $5.8(0.4)$ & 12,13 & $7.2(0.7)$ & 8,9 \\
Acuity improvement* & $4.7(0.5)$ & 0,1 & $-0.2(0.8)$ & $2,-2$ \\
Hypertension (\%) & 2 & 0 & 36 & 50 \\
Diabetes (\%) & 0 & 0 & 11 & 0 \\
Migraine (\%) & 6 & 50 & 6 & 0 \\
Acute onset (\%) & 43 & 0 & 67 & 50 \\
Disc oedema (\%) & 30 & 100 & 97 & 50 \\
Male (\%) & 35 & 50 & 65 & 50 \\
Pain (\%) & 77 & 0 & 11 & 50 \\
Field defect & & & & 0 \\
Altitudinal (\%) & 12 & 0 & 69 & 100 \\
Central (\%) & 67 & 0 & 17 & 0 \\
Other (\%) & 17 & 100 & 8 & \\
\hline
\end{tabular}

$\mathrm{ON}=$ optic neuritis; $\mathrm{AION}=$ anterior ischaemic optic neuropathy.

Distributions expressed as mean (SEM).

$\star$ Expressed in Snellen chart lines.

Training phase

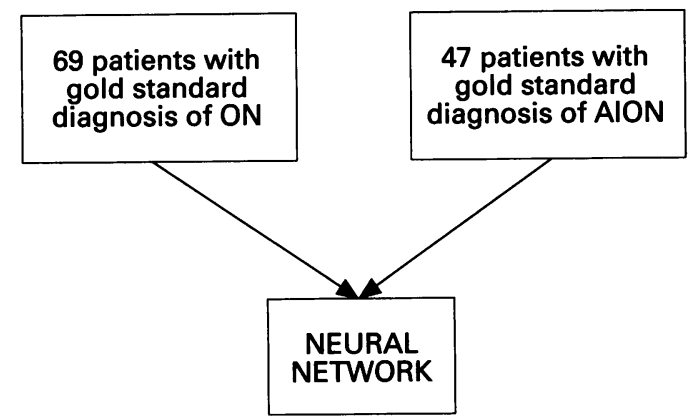

Testing phase

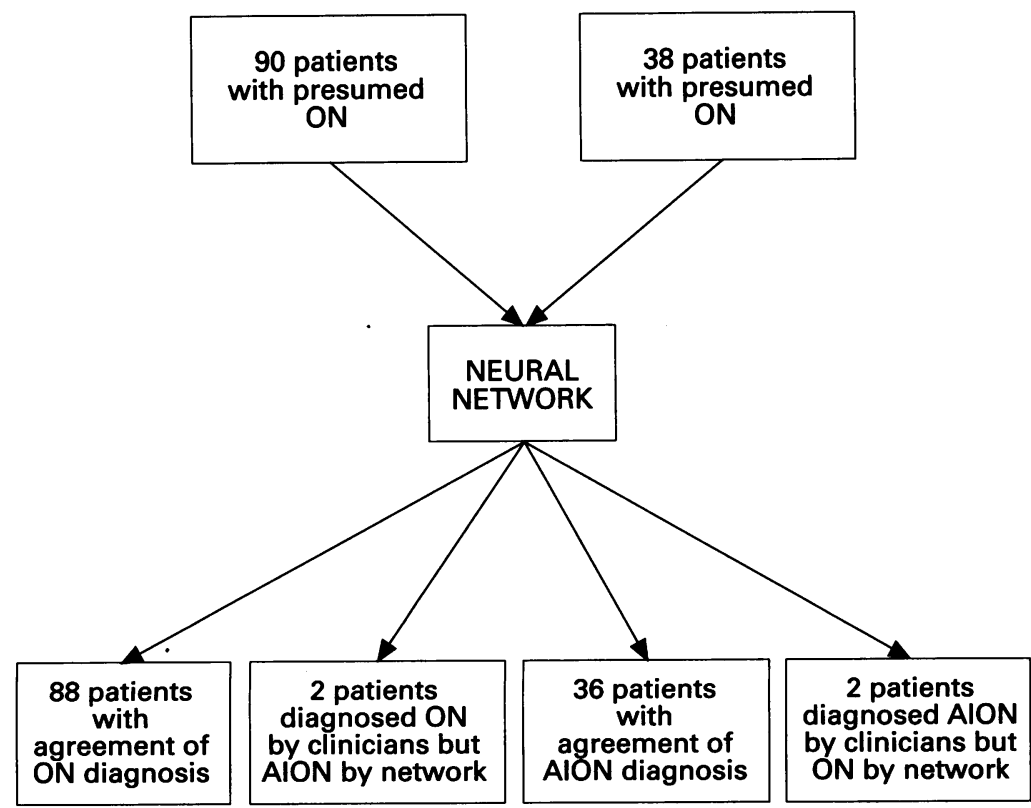

Figure 2 Flowchart specifying the groups of patients analysed by the neural network. In the training phase, the network was taught to recognise optic neuritis (ON) and anterior ischaemic optic neuropathy (AION) by training it with 116 'gold standard'patients (69 with $O N$ and 47 with $A I O N)$. In the testing phase, the accuracy of the network's diagnoses was determined using a separate group of 128 patients (90 presumed to have ON and 38 with AION). Unlike the gold standard patients used for training, the clinical diagnosis of the testing group was based purely on clinical impression at the time of the initial examination. clinical variable, while holding all others constant, and recording the effect on the predicted diagnosis. As a baseline, a theoretical patient was modelled so as to have equal predicted probabilities of $\mathrm{ON}$ and AION. Sequentially, each of the clinical criteria was changed from this baseline case, and the resulting network diagnosis determined.

\section{VALIDITY ANALYSIS}

To assess the accuracy of the training process with the gold standard patients, a validation analysis was done. Specifically, the gold standard patients were divided into four equal sized sets, and the network trained with data from three of them. The error rate of the network was then calculated when processing the data from the fourth set. This process was then repeated three more times, each time using a different set for testing.

DECISION ANALYTICAL MEASURES

Sensitivity of diagnosing ON was calculated as the number of true positive (concordant for $\mathrm{ON}$ ) diagnoses divided by the total number of patients clinically diagnosed with $O N$. Specificity of diagnosing ON was calculated as the number of true negative (concordant for AION) diagnoses divided by the total number of patients without $\mathrm{ON}$ (that is, clinically diagnosed with AION). Positive predictive value for diagnosing $\mathrm{ON}$ was calculated as the number of true positive diagnoses divided by the sum of the true positive and false positive cases.

\section{HIDDEN LAYER VARIATIONS}

To assess the relative contribution of the hidden layer (see Fig 1) of the neural network, the number of neurons in that layer was varied from 1 to 32 by multiples of 2 . For each size of the hidden layer, learning with the training data and testing on the test data were repeated completely, and the results recorded.

\section{Results}

This study of the accuracy of the neural network in differentiating $\mathrm{ON}$ and AION took place in two phases. Firstly, the neural network was trained with data from patients with gold standard diagnoses of $\mathrm{ON}$ and AION. Secondly, it was tested with data from a separate group of patients for whom the diagnosis was not as obvious. The error rates in this second phase were analysed, and compared with expert clinicians.

Specifically, in the first (training) phase, the network was presented in random order with the data from 116 patients with gold standard ON or AION. After this was repeated 342 times, or almost $40000(116 \times 342=39672)$ distinct training episodes, the network had learned these gold standard cases well enough so that its error rate was minimised. In other words, there was complete internal consistency in making the diagnoses (that is, no errors) when the network was retested with the data with which it was trained.

To further validate the training process, the patients from the gold standard diagnosis groups were subdivided into four sets, and a 
Table 3 Importance of clinical factors for neural network diagnosis of optic neuritis (ON) or anterior ischaemic optic neuropathy (AION)

\begin{tabular}{llll}
\hline Variable & Value & Activation $O N$ & Activation AION \\
\hline Idealised subject & Age 40 years & 0.500 & 0.500 \\
Elderly & Age 70 years & 0.002 & 0.998 \\
Young & Age 20 years & 0.988 & 0.012 \\
Male & & 0.116 & 0.884 \\
Female & & 0.883 & 0.117 \\
Hypertension & & 0.015 & 0.985 \\
Diabetes & & 0.356 & 0.644 \\
Migraine & 0.260 & 0.740 \\
Good initial acuity & $20 / 25$ & 0.860 & 0.150 \\
Poor initial acuity & $20 / 100$ & 0.190 & 0.810 \\
Altitudinal defect & & 0.044 & 0.956 \\
Central scotoma & & 0.948 & 0.052 \\
Other field defect & & 0.545 & 0.455 \\
Disc oedema & & 0.002 & 0.998 \\
Large improvement & 10 lines & 0.755 & 0.245 \\
No improvement & 0 lines & 0.038 & 0.962 \\
Progressive disease & & 0.801 & 0.199 \\
\hline
\end{tabular}

network trained with data from three of the sets. The network was then tested with data from the fourth set, and the error rates determined. This was repeated four times, so that each set could be tested. In the entire process a single error was made by the network in 116 trials, equal to a $99.1 \%$ accuracy rate.

In the second (testing) phase, attention was turned to testing the previously trained network on data from patients in whom the diagnosis was less clear. The 128 cases of presumed (not gold standard) $\mathrm{ON}$ and AION were presented serially to the previously trained network, and error rates calculated. In 117 cases $(91.4 \%)$ a highly confident diagnosis of either ON or AION was made, manifested by an output value greater than 0.99 (maximum being 1.0). The agreement of the network with the experienced clinicians is presented in Table 2 . Of the patients with presumed $\mathrm{ON}, 97.8 \%$ (88 of 90 ; groups I and II) were similarly diagnosed by the network, demonstrating strong concordance between clinicians and computer. Similarly, $94.7 \%$ ( 36 of 38 ; groups III and IV) of the patients with clinical AION were given that diagnosis by the network. Stated differently, the network had a $97.8 \%$ sensitivity and $94.7 \%$ specificity for diagnosing $\mathrm{ON}$. The positive predictive value of the network with respect to ON was $97.8 \%$.

The groups where the network and the clinician agreed on the diagnosis of ON and AION ('concordant' groups; groups I and III) were compared with those where there was disagreement ('discordant' groups; groups II and IV). These data are summarised in Table 2. The small numbers of patients in the discordant groups precluded statistical analysis. The patients in the concordant and discordant groups for each clinical diagnosis had roughly similar ages. Whereas only $30 \%$ of the patients in the ON concordance group (group I) had disc oedema, both patients in the group which the network predicted to have AION (group II) had disc oedema. This was similar to the $97 \%$ frequency of disc oedema in the AION concordance group (group III). The patients incorrectly diagnosed by the network to have AION (group II) had severe initial acuity loss, little improvement, no pain, and did not have central scotomas or altitudinal field defects. The patients incorrectly diagnosed by the net- work to have ON (group IV) were similar to those who actually had AION, except that they both had central scotomas.

The importance of each of the clinical criteria was assessed by tabulating the network predictions while each of the clinical variables was systematically varied (Table 3 ). All values were compared with an idealised patient for whom there was an equal probability of $O N$ and AION. Relative youth, female sex, better initial acuity, presence of a central scotoma, subsequent improvement in acuity, or progressive disease all biased the network towards a diagnosis of ON. Advanced age, male sex, presence of hypertension, poor initial acuity, an altitudinal field defect, disc oedema, or less improvement in acuity biased the network toward a diagnosis of AION.

The effect of varying the size of the hidden layer of the neural network was studied, using values of $1,2,4,8,16$, and 32 neurons in the layer. There was an inverse relation between the size of the hidden layer and the number of trials necessary for training to take place (Fig 3). There was little difference in the accuracy of the network across different hidden layer sizes (range 96.7-97.8\%).

\section{Discussion}

Neural networks have been used as artificial intelligence tools for modelling functions of the brain, including the visual and oculomotor systems. ${ }^{45}$ They have also been used as aids for diagnosis or classification, in areas as diverse as karyotyping banded chromosomes, ${ }^{6}$ differentiating the histological features of neoplasms, ${ }^{7}$ diagnosing myocardial infarction from enzyme data, ${ }^{8}$ analysing positron emission tomography scans for features typical of Alzheimer's disease, ${ }^{9}$ and distinguishing glaucomatous from non-glaucomatous automated visual fields. ${ }^{2}$ Neural networks are capable of distinguishing groups based on a wide variety of input variables, without a priori knowledge of which features are important in the input data. There is theoretical evidence that neural

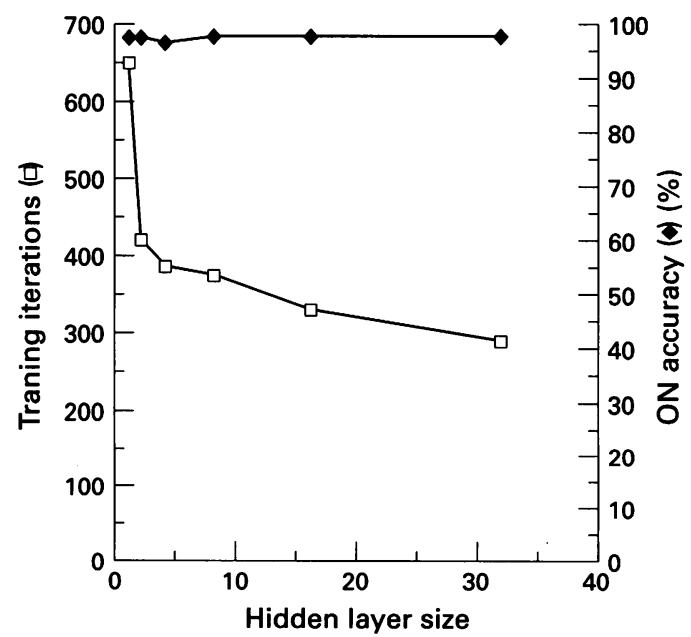

Figure 3 Effect of varying the number of neurons in the hidden layer. This was studied by calculating the diagnostic accuracy (filled diamonds, right axis) and number of repetitions required for training the network (open squares, left axis) for networks of $1,2,4,8,16$, and 32 hidden layer neurons. 
networks work at least as well as more conventional statistical methods for distinguishing groups of data, ${ }^{11}{ }^{11}$ if not better. ${ }^{1213}$

Because the neural network method is a powerful one for classifying groups, we studied its applicability to distinguish optic neuropathies which overlap clinically. ${ }^{1}$ We used data from our retrospective study of patients with either ON or AION, and found that the network had a high agreement with experienced clinicians in distinguishing the two optic neuropathies. The fact that the neural network almost completely distinguished the gold standard patients, even when trained on a subset of these patients and tested on another subset (validation analysis), suggests that there are reliable features that separate these patients.

The importance of each clinical feature in distinguishing the two groups was examined by varying the input from each feature in turn. The data from this analysis (Table 3) confirm the general teaching that $\mathrm{ON}$ is more likely to be associated with youth, female sex, central scotoma, and good improvement in acuity, whereas AION is more likely to be associated with advanced age, altitudinal field defect, disc oedema, and less improvement. Interestingly, initial good acuity was more likely to be associated with $\mathrm{ON}$, and poor initial acuity with AION. This finding was not evident in our earlier report, probably because acuity was analysed as a univariate factor while the neural network takes covarying factors into account. Whether this reflects population differences or simply selection bias in a referral population cannot be determined.

The clinicians and the network were not always in agreement. The network made the same diagnoses as the clinicians in $97.8 \%$ of the patients with $\mathrm{ON}$ and $94.7 \%$ of the patients with AION; two patients in each group had discordant diagnoses. There are at least two ways to explain the discrepant diagnoses found in these discordant groups. One possibility is that the clinicians were correct, and the network erroneously extrapolated data from the gold standard groups. Alternatively, the clinicians may have been incorrect. The two patients incorrectly diagnosed by the network with AION were middle aged, had subacute onset of severe visual loss, disc oedema, no pain, and did not improve over time. Many of these features are characteristic of AION, except for the age and tempo of visual loss. It is therefore debatable whether these patients truly had ON or AION. The two patients incorrectly diagnosed by the network with $\mathrm{ON}$ were similar to the patients with AION, but had central scotomas, a classic field defect in ON. One of these patients was 79 years old, and his acuity slightly worsened over time. If this patient actually had $\mathrm{ON}$, then the relative lack of improvement in acuity is consistent with reports of a decreased tendency of elderly patients with multiple sclerosis to recover function. ${ }^{14} 15$

The lack of correlation of hidden layer size and diagnostic accuracy might suggest that much of the information needed to discriminate the two groups does not require a hidden layer, and therefore is of low complexity. However, the non-linearity of the neural net neurons themselves has been shown to improve classification ability (compared with traditional techniques like linear discriminant analysis), possibly explaining the less critical importance of the hidden layer size. ${ }^{12}$

Neural network analysis cannot tell whether it was the clinician or the network that made the correct diagnosis. In fact, we were surprised to find that this technique had such a high sensitivity and specificity in distinguishing two overlapping optic neuropathies. However, our goal in using the neural network was not simply to simulate clinical diagnosis, but to investigate in an unbiased fashion the role of a neural network in distinguishing optic neuropathies that clinically overlap. The neural network methodology may therefore result in findings not otherwise derived from clinical experience. For example, from our analysis we can hypothesise that AION may be underdiagnosed in patients with subacute optic neuropathies, while $\mathrm{ON}$ may be underdiagnosed in older patients.

In summary, we have shown that the artificial intelligence technique of neural network analysis may aid in differentiating two common optic neuropathies. Similar methodologies may be useful in differentiating other clinically heterogeneous disorders, such as other optic neuropathies, motility disturbances, and the uveitides.

The financial support of the Heed Foundation (to LAL) and a departmental grant from Research to Prevent Blindness, Inc are gratefully acknowledged. The authors thank Dr Morton Smith for a critical reading of the manuscript.

1 Rizzo JF, Lessell S. Optic neuritis and ischemic optic neuropathy: overlapping clinical profiles. Arch Ophthalmol neuropathy: overlap

2 Goldbaum MH, Sample PA, White H, Côte B, Raphaelian $\mathrm{P}$, Fechtner $\mathrm{RD}$, et al. Interpretation of automated perimetry for glaucoma by neural network. Invest Ophthalmol Vis Sci 1994;35:3362-73.

3 McClelland JL, Rumelhart DE. Explorations in parallel distributed processing: explorations in the microstructure of cognition. Cambridge, MA: MIT Press, 1989.

4 Nawrot $M$, Blake R. A neural network model of kinetic depth. Vis Neurosci 1991;6:219-27.

5 Arnold DB, Robinson DA. A learning network model of the neural integrator of the oculomotor system. Biol Cybern 1991;64:447-54.

6 Graham J, Errington P, Jennings A. A neural network chromosome classifier. $\mathcal{F}$ Radiat Res (Tokyo) 1992; 33:250-7.

7 O'Leary TJ, Mikel UV, Becker RL. Computer-assisted image interpretation: use of a neural network to differentiate tubular carcinoma from sclerosing adenosis. Mod Pathol 1992;5:402-5.

8 Furlong JW, Dupuy ME, Heinsimer JA. Neural network analysis of serial cardiac enzyme data. A clinical application of artificial machine intelligence. Am $\mathcal{f}$ Clin Pathol 1991;96: $134-41$.

9 Kippenhan JS, Barker WW, Pascal S, Nagel J, Duara R. Evaluation of a neural network classifier for PET scans of normal and Alzheimer's disease subjects. $\mathcal{F}$ Nucl Med 1992; 33:1459-67.

10 Bourlard H, Kamp Y. Autoassociation by multilayer perceptrons and singular value decomposition. Biol Cybernetic 1988;59:291-4.

11 Gallinari P, Thiria S, Badran F, Fogelman-Soulie F. On the relations between discriminant analysis and multilayer perceptrons. Neural Networks 1991;4:349-60.

12 Reibnegger G, Weiss G, Werner-Felmayer G, Judmaier G Wachter $H$. Neural networks as a tool for utilizin laboratory information: comparison with linear discriminant analysis and with classification and regression trees. Proc Natl Acad Sci USA 1991;88:11426-30.

13 Astion ML, Wilding P. Application of neural networks to the interpretation of laboratory data in cancer diagnosis. Clin Chem 1992;38:34-8.

14 Hooge JP, Redekop WK. Multiple sclerosis with very late onset. Neurology 1992;42:1907-10.

15 Noseworthy J, Paty D, Wonnacott T, Feasby T, Ebers G. Multiple sclerosis after age 50 . Neurology 1983;33:153744. 